\title{
El cambio de la política migratoria México-Estados Unidos del 2018 al 2021
}

\section{Changes in the migratory policy of Mexico and the United States in the 2018-2021 period}

\author{
Alicia GutiérRez GonZÁLEZ \\ Universidad Anáhuac México, Facultad de Estudios Globales, México \\ alicia.gutierrez@anahuac.mx \\ https://doi.org/10.36105/iut.2021n34.02
}

Recibido: 09/09/2021

Aceptado: 18/11/2021

\section{RESUMEN}

El presente artículo tiene por objeto dar una visión general del cambio de la política migratoria México-Estados Unidos del 2018 al 2021. Este periodo se ha caracterizado por dos presidencias en los Estados Unidos: (i) la de Donald Trump (2017-2021) del Partido Republicano y (ii) la de Joe Biden desde el 20 de enero de 2021 del Partido Demócrata.

Palabras clave:

política migratoria, derechos humanos, migración irregular.

Abstract:

The purpose of this article is to provide an overall perspective regarding the changes of the Mexico- United States Migration Policy in the 2018-2021 period. This period has been defined by two presidential administrations in the United States: Donald Trump, backed by the GOP in the 2017-2021 term, and Joe Biden's, since January 20th, 2021. 
KeyWords:

Migratory Policy, Human Rights, irregular migration.

\section{Introducción}

El presente artículo tiene por objeto dar una visión general del cambio de la política migratoria México-Estados Unidos del 2018 al 2021. Este periodo se ha caracterizado por dos presidencias en los Estados Unidos: (i) la de Donald Trump (2017-2021) del Partido Republicano y (ii) la de Joe Biden desde el 20 de enero de 2021 del Partido Demócrata. La primera, trajo consigo el endurecimiento de la política migratoria (debido al aumento de solicitudes de asilo en su frontera sur) y la polarización de la sociedad debido a la retórica antiinmigrante y xenófoba. La segunda, desmanteló los Protocolos de Protección al Migrante (MPP, por sus siglas en inglés), también conocido como Quédate en México, auspiciada por Trump, que obligaba a los solicitantes de asilo a permanecer en México mientras se resolvía su solicitud. El artículo se divide en dos partes y la primera menciona brevemente: (i) la Declaración Conjunta celebrada entre México y Estados Unidos el 7 de junio de 2019 en relación con la Ley de Inmigración y Nacionalidad, sección 235 (b) (2) (C) de los Estados Unidos, MPP; (ii) los acuerdos y convenios firmados con los países del Triángulo del Norte (El Salvador, Guatemala y Honduras) relacionados con el asilo y el trabajo temporal; y (iii) la terminación del programa Quédate en México del 1 de junio de 2021.

La segunda parte explica: (i) el Plan Integral de Desarrollo en Centroamérica; (ii) estadísticas de la Comisión Mexicana de Ayuda a Refugiados (COMAR) del 2018 al 2021; (iii) menciona brevemente los derechos de los migrantes establecidos en la Ley sobre Refugiados, Protección Complementaria y Asilo Político del 2011; (iv) indica el procedimiento para solicitar asilo en México ante la COMAR; (v) menciona algunas de las tarjetas que expide el Instituto Nacional de Migración (INM) para los diferentes tipos de migrantes que así lo soliciten; y comenta el memorándum de entendimiento de cooperación internacional celebrado el 8 de junio de 2021 entre la Secretaría de Relaciones Exteriores de México y la Agencia de Estados Unidos para el Desarrollo Internacional, cuya intención es lograr prosperidad, equidad y seguridad en Norteamérica.

\section{El endurecimiento de la política estadounidense}

\section{La Declaración Conjunta México-Estados Unidos de 7 de junio de 2019}

Para un mayor entendimiento de lo que significa la Declaración Conjunta México-Estados Unidos del 7 de junio del $2019^{1}$ hay que conocer los antecedentes. Con fecha 20 de diciembre de 2018, los Estados Unidos anunciaron los

\footnotetext{
Secretaría de Relaciones Exteriores, “Declaración Conjunta de 7 de junio de 2019”, https://www.gob.mx/sre/ documentos/declaracion-conjunta-203701.
} 
Protocolos de Protección al Migrante (MPP, por sus siglas en inglés), también conocido como el programa Quédate en México, que obligaba a los solicitantes de asilo a permanecer en México mientras se resolvía su solicitud. Cabe hacer mención que el gobierno mexicano confirmó el mismo día dicho anuncio indicando que por razones humanitarias, autorizaría ese protocolo. Resulta pertinente mencionar que durante el 2018 y el 2019, caravanas de migrantes de Centroamérica y Cuba cruzaron por México con la finalidad de llegar a los Estados Unidos para solicitar asilo por diversos motivos. Las caravanas centroamericanas que llegaron a Tijuana entre noviembre y diciembre de 2018 estaban conformadas por casi 10 mil personas, la caravana que llegó a Piedras Negras, Coahuila en febrero de 2019 estaba integrada por alrededor de 1850 personas y la que llegó a Ciudad Juárez entre octubre de 2018 y abril de 2019 estaba compuesta por más de 15000 personas. $^{2}$ Esta nueva forma de emigrar tomó por sorpresa tanto a los gobiernos como a la sociedad civil. Ahora bien, la idea de viajar en caravanas surgió por la necesidad de sentir una mayor protección y así evitar abusos y posibles desapariciones forzadas. Además, al viajar en caravana, el costo del viaje disminuye porque se logra captar la atención de los gobiernos y de las organizaciones no gubernamentales. ya sean civiles o religiosas que se encargan de ayudarlos y de darles alojamiento, comida y asesoría. Es importante mencionar que no todos los migrantes dejan su lugar de origen por inseguridad o discriminación por razón de raza u orientación sexual. También existen los migrantes económicos que buscan mejorar sus condiciones de vida y la reunificación familiar.

Una vez explicado lo anterior, cabe destacar que el entonces secretario del Departamento de Seguridad Nacional (DHS, por sus siglas en inglés) Kirstjen Nielsen emitió un memorándum el 25 de enero de 2019 titulado "La Orientación sobre Políticas para la Implementación de los Protocolos de Protección al Migrante". ${ }^{3}$ Ahí se menciona que el programa Quédate en México se implementaría para abordar la crisis migratoria a lo largo de la frontera sur. En mayo del 2019 se registraron en la frontera estadounidense 144,116 detenciones. ${ }^{4}$ Lo anterior trajo como consecuencia que el gobierno del expresidente Donald Trump endureciera la política migratoria. Ante dicho incremento y con la xenofobia y retórica antiinmigrante que le caracterizó durante todo su mandato, éste declaró, qué si no se reducían las llegadas de los solicitantes de asilo a su país, entonces se vería en la necesidad de aplicar una nueva política arancelaria en contra de las importaciones mexicanas. ${ }^{5}$ En consecuencia, el 7 de junio del 2019 México y

2 Contreras Delgado, Camilo, París Pombo, María Dolores y Velasco Ortiz, Laura (coords.), Caravanas Migrantes y Desplazamientos Colectivos en la Frontera México-Estados Unidos, México, El Colegio de la Frontera Norte, 2021, p. 18.

3 Departamento de Seguridad Nacional, “Orientación sobre Políticas para la Implementación de los Protocolos de Protección al Migrante" (Policy guidance for implementation of the Migrant Protection Protocols), https:// www.dhs.gov/sites/default/files/publications/19_0129_OPA_migrant-protection-protocols-policy-guidance.pdf.

4 Aduanas y Protección fronteriza de Estados Unidos, "Detenciones en la frontera", en: la Razón, 7 de enero de 2020, p. 7.

5 De HaAs Hein, Castles Stephen y Miller, Mark, The age of migration: International population movements in the modern world, sexta edición, Nueva York, Guilford Publications, 2020, p. 169. 
Estados Unidos firmaron una Declaración Conjunta ${ }^{6}$ de la que se desprendía que en el plazo de 90 días el gobierno mexicano se comprometía a frenar los flujos migratorios. En el rubro de la instrumentación de la sección 235(b)(2)(c) de la Declaración se establece que:

los Estados Unidos extenderán de manera inmediata la instrumentación de la sección 235 (b) (2) (C) a lo largo de su frontera sur. Ello implica que aquellos que crucen la frontera sur de Estados Unidos para solicitar asilo serán retornados sin demora a México, donde podrían esperar la resolución de sus solicitudes de asilo.

A su vez, por razones humanitarias y en cumplimiento de sus obligaciones internacionales, autorizará la entrada de dichas personas mientras esperan la resolución de sus solicitudes de asilo. México de acuerdo con sus principios de justicia y fraternidad universales, ofrecerá oportunidades laborales y acceso a la salud y educación a los migrantes y sus familias mientras permanezcan en territorio nacional, así como protección a sus derechos humanos. Los Estados Unidos se comprometen a acelerar la resolución de solicitudes de asilo y proceder con los procedimientos de remoción lo más expedito posible.

Esta Declaración entre México y los Estados Unidos convirtió a México en un tercer país de facto, aunque no de iure, ya que todos los solicitantes de asilo fueron regresados a México mientras se resolvía su solicitud de asilo a los Estados Unidos. En mérito de lo anterior, se puede decir que tanto la política estadounidense como la retórica de Trump muestran una clara forma de securitización ${ }^{7}$ de la migración, en la que se muestra a los migrantes como una amenaza para la seguridad y la integridad cultural del país, lo que por desgracia ha fomentado el incremento del nacionalismo.

Ahora bien, en septiembre de 2019 el canciller mexicano Marcelo Ebrard dio a conocer qué entre junio y agosto del mismo año el flujo migratorio se había reducido en un $56 \% \%^{8}$ con lo que se logró que las medidas arancelarias que se habían dado a conocer fueran suspendidas. La reducción del flujo migratorio fue posible debido al endurecimiento de la política migratoria mexicana y al despliegue de la guardia nacional en la frontera sur de México.

Los migrantes que llegan a la frontera de Estados Unidos transitan seis rutas y las más comunes son las siguientes: ${ }^{9}$

(i) La ruta del norte que va de Querétaro a Ciudad Juárez $(1,719 \mathrm{~km})$;

(ii) La ruta del noroeste que va de Querétaro a Tijuana $(2,644 \mathrm{~km})$;

(iii) La ruta del sureste que va de Tenosique a Tierra Blanca $(665 \mathrm{~km})$;

(iv) La ruta del suroeste que va de Tapachula a Tierra Blanca $(684 \mathrm{~km})$;

(v) La ruta del centro que va de Tierra Blanca a Querétaro $(591 \mathrm{~km}) \mathrm{y}$;

(vi) La ruta del noreste que va de Querétaro a Ciudad Acuña (1,134 km).

\footnotetext{
Secretaría de Relaciones Exteriores, "Declaración Conjunta de 7 de junio de 2019", op. cit.

De Haas Hein, Castles Stephen y Miller, Mark, op. cit., pp. 169 y 232.

PINEDA, Perla, "Ebrard presentará informe migratorio" El Economista, 9 de septiembre de 2019, p. 44.

9 Nieto, Francisco, "Camino hacia EU”, El Heraldo de México, 19 de junio de 2019, https://issuu.com/ elheraldodemexico/docs/el_heraldo_771.
} 
En estas rutas existen casas de migrantes y albergues. Sin embargo, ante el aumento exponencial de los migrantes, estos albergues no han sido suficientes y con las restricciones estadounidenses, los migrantes que no quieren permanecer en México buscan rutas alternas ilegales, ya sean terrestres o marítimas. Lo anterior, aumenta significativamente el riesgo y costo para llegar a los Estados Unidos.

Cabe destacar que el programa Quédate en México se expandió a lo largo de la frontera norte de México y cuando inició la pandemia de COVID-19 (enfermedad causada por el SARS-CoV-2) en marzo del 2020, se cerró la frontera invocando el título $42^{10}$ por la emergencia sanitaria y por ende se suspendieron todas las inscripciones y casos bajo el MPP. En este orden de ideas, los Centros para el Control y la Prevención de Enfermedades de los Estados Unidos (CDC, por sus siglas en inglés) emitieron una orden bajo el título en mención, donde se establece la prohibición de la entrada al país de personas sin autorización, incluso de solicitantes de asilo. Como consecuencia, el CDC y el DHS declararon que ejecutarían una política de expulsión sumaria de personas detenidas tras cruzar la frontera sur de los Estados Unidos de manera irregular con fundamento en el multicitado título 42 por razones de salud pública. Esta política se extendió por tiempo indefinido y claramente atenta contra los derechos de los migrantes y solicitantes de asilo, ya que los indocumentados pueden ser expulsados de forma inmediata al país por el que ingresaron sin que medie un procedimiento legal de deportación. Lo anterior, viola el marco legal de los Estados Unidos, ya que so pretexto de la pandemia de COVID-19, se expulsaron migrantes de forma expedita.

\section{Los Acuerdos y Convenios con los países del Triángulo del Norte}

El Departamento de Seguridad de Estados Unidos también firmó acuerdos y convenios con los países del Triángulo del Norte ${ }^{11}$ (El Salvador, Guatemala y Hondura), ya que en el año fiscal del mes de agosto de 2019 más del 72\% de todos los migrantes arrestados en la frontera pertenecían a esos países. Así las cosas, de mayo a octubre de 2019 se llevaron a cabo con dichos países:

(i) Acuerdos de Cooperación de Asilo (ACA);

(ii) Convenios de seguridad fronteriza;

(iii) Convenios del Programa para intercambiar información biométrica (BDSP) y;

(iv) Acuerdos para programas de trabajadores temporales en actividades agrícolas y no agrícolas.

Los ACA consisten en permitir a los migrantes buscar protección dentro de la región al facilitar la cooperación entre Estados Unidos y los gobiernos de los

10 U.S. Customs and Border Protection, "Nationwide Enforcement Encounters: Title 8 Enforcement Actions and Title 42 Expulsions", https://www.cbp.gov/newsroom/stats/cbp-enforcement-statistics/title-8-and-title-42statistics.

11 Departamento de Seguridad Nacional de EUA, “Acuerdos del DHS con Guatemala, Honduras y El Salvador”, https://www.dhs.gov/sites/default/files/publications/19_1007_fact-sheet-spanish-agreements-w-northernregion-central-am-countries.pdf. 
países receptores $\mathrm{u}$ organismos internacionales a fin de expandir sus sistemas de protección humanitaria. El propósito de los Convenios de seguridad fronteriza es el despliegue de funcionarios del Servicio de Aduanas y Protección Fronteriza de Estados Unidos y del Servicio de Inmigración y Control de Aduanas de los Estados Unidos como consejeros y asesores de sus contrapartes en la policía, la seguridad fronteriza, inmigración y aduanas del país receptor. El objetivo de los Convenios del Programa para intercambiar información biométrica es mejorar la cooperación entre el DHS y los países del Triángulo del Norte a fin de prevenir y combatir el crimen y otras amenazas a la seguridad pública mediante una mayor recolección de datos biométricos y el intercambio de información. Finalmente, los Acuerdos para programas de trabajadores temporales en actividades agrícolas y no agrícolas se firmaron solamente con el Departamento de Trabajo de los Estados Unidos y Honduras y Guatemala y no con el DHS.

En la siguiente tabla se muestran los casos de asilo afirmativos presentados ante el Servicio de Ciudadanía e Inmigración de los Estados Unidos (USCIS, por sus siglas en inglés), de los años fiscales 2018 y 2019 respectivamente (el año fiscal inicia el primero de octubre y termina el 30 de septiembre).

Tabla 1: Casos de asilo afirmativo de los años fiscales 2018 y 2019

\begin{tabular}{|c|c|c|}
\hline País & $\mathbf{2 0 1 8}$ & $\mathbf{2 0 1 9}$ \\
\hline El Salvador & 9140 & 5951 \\
\hline Guatemala & 10192 & 9684 \\
\hline Honduras & 6143 & 5609 \\
\hline México & 6618 & 4588 \\
\hline
\end{tabular}

Fuente: Servicio de Ciudadanía e Inmigración de los Estados Unidos (USCIS, por sus siglas en inglés). ${ }^{12}$

\section{La terminación del programa Quédate en México o MPP}

El 20 de enero de 2021, el entonces secretario David Pekoske emitió un memorándum suspendiendo nuevas inscripciones al programa MPP. El 2 de febrero de 2021 el presidente Joe Biden emitió una orden ejecutiva bajo el número 14010, 86 Fed. Reg. 8267 y el Departamento de Seguridad Nacional de los Estados Unidos anunció el 11 de febrero de 2021 el plan ${ }^{13}$ para continuar con el procesamiento a Estados Unidos de las personas ya registradas en el MPP y que fueron devueltos a México, así como a los que tienen casos pendientes ante la Oficina Ejecutiva para la Revisión de Casos de Inmigración (EOIR, por sus siglas en inglés). El plan comenzó el 19 de febrero, por lo que el DHS inició con la

\footnotetext{
12 Baugh, Ryan, “Annual Flow Report, "Refugees and Asylees 2019, septiembre de 2020, https://www.dhs. gov/sites/default/files/publications/immigration-statistics/yearbook/2019/refugee_and_asylee_2019.pdf.

13 Departamento de Seguridad Nacional, "Anuncia proceso para atender a individuos en México con casos pendientes bajo MPP”, https://www.dhs.gov/news/2021/02/11/departamento-de-seguridad-nacional-dhsanuncia-proceso-para-atender-individuos-en-m\#.
} 
primera etapa para restablecer un proceso seguro y ordenado en la frontera $\mathrm{Su}-$ roeste. El DHS comentó en el plan mencionado con antelación que alrededor de 25,000 personas retornadas bajo el programa Quédate en México mantenían aún casos abiertos.

Ahora bien, el secretario de Seguridad Nacional, Alejandro N. Mayorkas señaló que existe el compromiso para reformar las políticas migratorias que no están alineadas con los valores de los Estados Unidos. Sin embargo, expresó qué debido a la pandemia, las restricciones en la frontera seguían vigentes. El número de extranjeros registrados bajo el MPP por la Oficina de Aduanas y Protección Fronteriza de Estados Unidos (CBP, por sus siglas en inglés) a lo largo de la frontera Suroeste fue de 68,039 a diciembre de 2020. ${ }^{14}$

Ahora bien, el 1 de junio del 2021 tras una revisión minuciosa del multicitado programa Quédate en México y en cumplimiento con el mandato del presidente Joe Biden vía Orden Ejecutiva 14010, el secretario del Departamento del DHS decidió finalizar el MPP. ${ }^{15}$

Así las cosas, Joe Biden está dando marcha a tras a las órdenes ejecutivas de Donald Trump relacionadas con el endurecimiento de la política migratoria estadounidense. Por lo tanto, existe mayor tranquilidad para quienes viven en los Estados Unidos bajo el estatus de protección temporal (TPS, por sus siglas en inglés) o bajo la Acción Diferida para los llegados en la Infancia (DACA, por sus siglas en inglés), conocidos como dreamers (soñadores en español). La reforma migratoria propuesta por Biden señala que precisamente esos sectores mencionados con antelación pueden aspirar directamente a la ciudadanía. Al respecto, hay que recordar que la ciudadanía en los Estados Unidos se obtiene por el ius soli, i.e., el derecho de suelo o el derecho a la nacionalidad de un Estado por el solo hecho de haber nacido en ese territorio. Sin embargo, la nacionalidad por naturalización también se puede adquirir bajo el principio de ius domicili o derecho de domicilio, después de haber radicado cinco años en el país. ${ }^{16}$

Se puede decir que con la llegada a la presidencia de Joe Biden, la retórica ha cambiado, sin embargo, el titulo 42 se sigue aplicando y la frontera continuará cerrada hasta por lo menos el 21 de julio del 2021 según el informe del DHS por los aumentos del COVID-19 en México en los últimos días de junio. ${ }^{17}$

Según los memorándums emitidos por el DHS, el enfoque estadounidense para los próximos meses será principalmente en seguridad nacional, fronteriza y pública.

14 Departamento de Seguridad Nacional, "Métricas y Medidas de los Protocolos de Protección a Migrantes", https://www.dhs.gov/sites/default/files/publications/metricas_y_medidas_de_los_protocolos_de_ proteccion_a_migrantes_2.pdf.

15 Departamento de Seguridad Nacional, "Terminación del Programa de Protección al Migrante”, 1 de junio de 2021, https://www.dhs.gov/sites/default/files/publications/21_0601_termination_of_mpp_program.pdf.

16 Koser, Khalid, International Migration. A very short introduction, 2a. ed., Oxford, Oxford University Press, 2016, p. 20.

17 Gutiérrez González, Alicia, "Finaliza programa quédate en México", El Sol de México, 22 de junio de 2021, https://www.elsoldemexico.com.mx/analisis/finaliza-programa-quedate-en-mexico-6872525.html. 
A continuación, se puede apreciar en la tabla, el aumento de cruces ilegales de mexicanos en los primeros trimestres del 2018 al 2021 y al hacer la comparación de los años anteriores, se observa un aumento exponencial en el primer trimestre del 2021.

Tabla 2: Cruce ilegal de mexicanos a los Estados Unidos de 2018-2021

\begin{tabular}{|c|c|}
\hline Primer trimestre de 2018 & 61,881 mexicanos \\
\hline Primer trimestre de 2019 & 56,790 mexicanos \\
\hline Primer trimestre de 2020 & 65,280 mexicanos \\
\hline Primer trimestre de 2021 & 147,394 mexicanos \\
\hline
\end{tabular}

Fuente: Pérez Maritza, en El Economista, 9 de abril de 2021. ${ }^{18}$

\section{La política migratoria y de asilo en México del 2018 al 2021}

La política migratoria y de asilo en México del 2018 al 2021 ha ido cambiando a través del tiempo. Jorge Durand comenta que la migración entre México y Estados Unidos ha sido una constante entre ambos países por la oferta y la demanda de mano de obra, ya que un país es rico en capital y pobre en mano de obra y el otro a la inversa. ${ }^{19}$ La migración se puede dividir en: (i) migración legal, (ii) ilegal o irregular; y (iii) forzada. Esta última se caracteriza por tener un elemento coercitivo, incluyendo en algunos casos, la amenaza a la vida y/o a la subsistencia y puede ser por causas naturales (desastres naturales o medioambientales) o por causas humanas (movimientos de refugiados y de población desplazada internamente, desastres nucleares o químicos, hambruna o proyectos de desarrollo).

México cuenta con una Ley sobre Refugiados, Protección Complementaria y Asilo Político. ${ }^{20}$ En su artículo quinto señala los principios y criterios en los que se fundamenta la ley en mención. El principio de no devolución, el principio de no discriminación, el principio de interés superior del niño y el principio de unificación familiar entre otros. Estos principios son muy importantes ya que se alinean al contexto internacional.

Al respecto, el principio de no devolución es medular para la protección de los refugiados, toda vez que después de la Segunda Guerra Mundial se crea el Alto Comisionado de las Naciones Unidas para los Refugiados (ACNUR) con la finalidad de proteger a los refugiados. En lo que concierne a México, esté se adhiere a la Convención de Ginebra de 1951 sobre el Estatuto de los Refugiados

18 PÉREZ, Maritza, "Crecen 125\% cruces hacia EU de migrantes mexicanos”, El Economista, 9 de abril de 2021, https://www.eleconomista.com.mx/politica/Crecen-125-cruces-hacia-EU-de-migrantes-mexicanos-202104090005.html.

19 Durand, Jorge, Historia mínima de la migración México-Estados Unidos, México, El Colegio de México, primera reimpresión, 2017, p. 13.

20 Ley sobre Refugiados, Protección Complementaria y asilo Político, del 27 de enero de 2011, http://www. diputados.gob.mx/LeyesBiblio/pdf/LRPCAP_111120.pdf. 
y del Protocolo de $1967^{21}$ el 7 de junio del año 2000, adhesión que lo obliga a respetar y a cumplir con lo estipulado en dicha Convención. Este principio previsto en el artículo 33 de la Convención en cita establece claramente la prohibición de expulsión y de devolución (Refoulement en francés) y señala que: "1. Ningún Estado Contratante podrá, por expulsión o devolución, poner en modo alguno a un refugiado en las fronteras de territorios donde su vida o su libertad peligre por causa de su raza, religión, nacionalidad, pertenencia o determinado grupo social o de sus opiniones políticas".

Ahora bien, el artículo 13 de la Ley en mención establece que las personas extranjeras que se encuentren en territorio mexicano pueden solicitar refugio en México si se encuentran grosso modo dentro de los siguientes supuestos: (i) temor a ser perseguido por motivos de raza; religión; nacionalidad; género; pertenencia a determinado grupo social u opiniones políticas; (ii) temor por su vida; su seguridad; o porque su libertad ha sido amenazada por violencia generalizada; agresión extranjera; conflictos internos; violación masiva de los derechos humanos, etcétera.

Los derechos y obligaciones de los refugiados se encuentran enumerados en el artículo 44 de la misma Ley y señala que si existe miedo por parte de los extranjeros de regresar a su país de origen, entonces se solicitará la protección por parte del Estado mexicano, quien se compromete a otorgarles a los refugiados el servicio de salud, educación, reunificación familiar, apoyo por parte de las instituciones públicas, así como a expedirles un documento que les acredite su legal estancia en el país.

Una vez que se ingresa al país, se tienen 30 días hábiles para presentar la solicitud de refugiado ante la Comisión Mexicana de Ayuda a Refugiados $(\mathrm{COMAR})^{22} \mathrm{o}$ ante las oficinas de regulación migratoria del Instituto Nacional de Migración (INM). Cuando se realiza la solicitud, se les entrega una constancia donde se establece que su situación migratoria se encuentra en trámite y por lo tanto no pueden ser devueltos a su país de origen, en tanto no se resuelva su situación. Con esa constancia, se solicita al INM una tarjeta de visitante por razones humanitarias. Esa tarjeta es gratuita, les permite trabajar, la vigencia es de un año y les ofrece una estancia regular en el país. Es necesario que en el formulario se expongan las razones por las que tuvieron que salir y dejar su país y el motivo por el que no pueden regresar. Una vez hecho lo anterior, la COMAR entrevista a los solicitantes y evalúa si efectivamente la vida del solicitante corre peligro e informa la resolución en 45 días hábiles. El plazo puede extenderse de 10 a 45 días o más, dependiendo del cúmulo de trabajo y del personal. Si la resolución es positiva, entonces, en coordinación con la COMAR hay que acudir al INM para tramitar la residencia permanente en México como refugiado. Si la resolución es negativa, entonces, se puede apelar en un plazo de 15 días. Presentada la apelación,

\footnotetext{
21 Convención de Ginebra de 1951 sobre el Estatuto de los Refugiados y del Protocolo de 1967, https://www. acnur.org/fileadmin/Documentos/BDL/2001/0506.pdf.

22 Comisión Mexicana de Ayuda a Refugiados, "Requisitos para solicitar Refugio en México", https://www.gob. $\mathrm{mx} /$ comar.
} 
la COMAR revisa el caso nuevamente en aproximadamente 90 días naturales. En caso negativo hay que retornar al país de origen.

Cabe mencionar que para hacer frente al fenómeno migratorio el INM expide diversas tarjetas entre las que destacan:23 (i) la Tarjeta de Residente Temporal (TRT); (ii) la Tarjeta de Residente Permanente (TRP); (iii) la Tarjeta de Visitante Regional (TVR) y; (iv) la Tarjeta de Visitante Trabajador Fronterizo (TVTF). El cambio por ejemplo de la TRT a TRP se obtiene cuando un extranjero con condiciones de estancia de residente temporal cumplió cuatro años consecutivos de residencia. La TVR, por su parte, permite a los extranjeros de origen guatemalteco o beliceño ingresar por tiempo limitado a la frontera sur de México de manera fácil y segura. Con la TVTF los guatemaltecos o beliceños que cuentan con alguna invitación laboral en México pueden entrar y salir en múltiples ocasiones en los estados de la frontera sur (Campeche, Chiapas, Tabasco y Quintana Roo). La visa por razones humanitarias se otorga al extranjero que ha sido víctima de catástrofe natural o violencia, cuya vida o integridad se encuentre en peligro o bien que ingrese para realizar acciones de auxilio o rescate en situaciones de emergencia en el país. La visa por unidad familiar ayuda a los extranjeros que viven en el país y desean solicitar visa para sus familiares del exterior.

Estadísticas de la Comisión Mexicana para Ayuda a Refugiados del 2018 al 2021

A continuación, se muestran varias estadísticas de la COMAR del 2018, ${ }^{24}$ 2019, 2020 y $2021^{25}$ relacionados con los solicitantes de asilo en México. En el 2018 se recibieron 29,630 solicitudes, en el 2019 aumentaron a 70,418, en el 2020 por la pandemia se redujeron a 41,179 y hasta el mes de mayo de 2021 van 41,195. Esto es claramente consecuencia del programa Quédate en México. Afortunadamente, este programa se dio por terminado el primero de junio de 2021.

Tabla 3: Solicitantes de asilo por nacionalidad (personas) del 2018, 2019, 2020 y 2021

\begin{tabular}{|l|c|r|r|c|}
\hline \multicolumn{1}{|c|}{ País } & $\mathbf{2 0 1 8}$ & $\mathbf{2 0 1 9}$ & $\mathbf{2 0 2 0}$ & 2021 (mayo) \\
\hline Brasil & ----- & 554 & 367 & 439 \\
\hline Camerún & ----- & 512 & ---- & ---- \\
\hline Colombia & 210 & 559 & 498 & 413 \\
\hline Cuba & 214 & 8,686 & 5,749 & 3,769 \\
\hline Chile & ----- & ---- & 805 & 866 \\
\hline
\end{tabular}

23 Instituto Nacional de Migración, “Trámites Migratorios”, https://www.gob.mx/inm/acciones-y-programas/ tramites-migratorios.

24 COMAR, "Estadísticas de solicitantes de la condición de refugiado en México", marzo de 2020, https://www. gob.mx/comar/articulos/estadisticas-de-solicitantes-de-la-condicion-de-refugiado-en-mexico.

25 COMAR, "La COMAR en números", mayo de 2021, https://www.gob.mx/comar/articulos/la-comar-ennumeros-273823?idiom=es. 


\begin{tabular}{|l|r|r|r|r|}
\hline El Salvador & $\mathbf{6 , 1 9 3}$ & $\mathbf{9 , 0 4 3}$ & $\mathbf{4 , 0 5 0}$ & $\mathbf{3 , 0 0 8}$ \\
\hline EE.UU. & 35 & ----- & ---- & ------ \\
\hline Guatemala & $\mathbf{1 , 3 4 7}$ & $\mathbf{3 , 7 8 3}$ & $\mathbf{3 , 0 0 5}$ & $\mathbf{1 , 6 1 5}$ \\
\hline Haití & 76 & 5,539 & 5,950 & 7,135 \\
\hline Honduras & $\mathbf{1 3 , 6 7 9}$ & $\mathbf{3 0 , 1 0 4}$ & $\mathbf{1 5 , 4 6 5}$ & $\mathbf{1 9 , 0 3 3}$ \\
\hline Nicaragua & 1,271 & 2,233 & 802 & 936 \\
\hline Venezuela & 6,326 & 7,634 & 3,273 & 2,063 \\
\hline Yemen & 29 & ----- & ----- & ------ \\
\hline Otros países & 250 & 1,771 & 1,205 & 1,164 \\
\hline Total & $\mathbf{2 9 , 6 3 0}$ & $\mathbf{7 0 , 4 1 8}$ & $\mathbf{4 1 , 1 7 9}$ & $\mathbf{4 1 , 1 9 5}$ \\
\hline
\end{tabular}

Fuente: Elaboración propia con información de la COMAR del 2018 al corte de mayo del 2021.

En la tabla anterior se aprecia como los países del Triángulo del Norte siguen siendo los principales solicitantes de asilo en México. Esto se debe por una parte a la pandemia por COVID-19, y a la inseguridad y a la falta de oportunidades en sus países de origen por la otra. Como se mencionó con anterioridad, es muy notorio el incremento de las solicitudes en el 2019 debido al MPP.

\section{El Plan Integral de Desarrollo para Centroamérica}

Al ser México un país de origen, tránsito, destino y retorno de migrantes, el gobierno mexicano firmó una Declaración Conjunta con El Salvador, Guatemala, y Honduras con el apoyo y acompañamiento de la CEPAL alineado con el Pacto Mundial para atender las causas estructurales de la migración más allá de sus fronteras. El Canciller mexicano Marcelo Ebrard señaló que México está comprometido con Centroamérica desde una perspectiva regional e integral y facilitará una vecindad basada en la solidaridad y la prosperidad, impulsando la cooperación y los Objetivos de Desarrollo Sostenible de la Agenda $2030^{26}$ con atención a todo el ciclo migratorio bajo el principio de responsabilidad compartida, pero diferenciada, con pleno respeto y protección de los derechos humanos sin importar la condición migratoria. ${ }^{27}$

México firmó el Pacto Mundial sobre los Refugiados ${ }^{28}$ para una Migración Segura, Ordenada y Regular. Aunque el Pacto no es jurídicamente vinculante muestra la voluntad política de la comunidad internacional para fortalecer la

26 Agenda 2030, "La Asamblea General adopta la Agenda 2030 para el Desarrollo Sostenible", https://www. un.org/sustainabledevelopment/es/2015/09/la-asamblea-general-adopta-la-agenda-2030-para-el-desarrollosostenible/.

27 Secretaría de Relaciones Exteriores, "Intervención del secretario de Relaciones Exteriores de México, Marcelo Ebrard. Conferencia Intergubernamental para la adopción del Pacto Mundial para la Migración Segura, Ordenada y Regular, Sesión plenaria”, Marruecos, 10 de diciembre de 2018, https://www.un.org/en/conf/ migration/assets/pdf/GCM-Statements/mexico.pdf.

Alto Comisionado de las Naciones Unidas para los Refugiados (ACNUR), Pacto Mundial sobre Refugiados, con una nota introductoria de la Oficina del Alto Comisionado de las Naciones Unidas para los Refugiados (ACNUR), 17 de diciembre de 2018, https://www.refworld.org.es/docid/5c781b294.html. 
cooperación y la solidaridad con los países de acogida, que resultan ser los más afectados en esta era del éxodo migrante. El objetivo medular de este pacto es implementar el principio de distribución de la carga y el principio de responsabilidad, sustentado también en el principio de no devolución constituido por la Convención de Ginebra de 1951 y su Protocolo de 1967, principio adoptado por la legislación mexicana. El pacto busca la cooperación de los países para aprovechar los beneficios de la migración y en el caso de Centroamérica, mejorar las condiciones en el Triángulo del Norte y fortalecer la cooperación internacional y crear alianzas mundiales para una migración segura, ordenada y regular. Los 23 objetivos del Pacto se centran en la protección de los derechos humanos, la inclusión y la cohesión social.

El Plan Integral de Desarrollo surge por el aumento en las migraciones a México y por ende a los Estados Unidos a causa de la inseguridad que se vive en Centroamérica y en los países del Triángulo del Norte. Muchas personas han tenido que dejar sus países en busca de nuevas oportunidades. Los principales factores por los que los individuos emigran se pueden dividir en tres: (i) los sociales; (ii) los económicos; y (iii) los medioambientales. En los primeros, se encuentran la separación familiar; la violencia; la extorsión; los conflictos bélicos y discriminación por razón de sexo u orientación sexual, etc. En los segundos, el endeudamiento; el desempleo; los salarios bajos; la dependencia del trabajo agrícola; etc. Y en los terceros, se tienen los medioambientales que surgen por la variabilidad climática, la erosión del suelo y los desastres naturales.

Así las cosas, como ya se comentó con anterioridad, durante el 2018 y el 2019 surgió el fenómeno de la migración en caravana, cuyo objetivo principal era contar con protección y seguridad que un grupo social puede brindar. Ahora bien, el futuro al que se enfrentan los migrantes en el paso por México hacia los Estados Unidos es la invisibilidad, ya que miles de Centroamericanos arriesgan su vida y son expuestos a la violencia, la extorsión, la desaparición forzada, y al crimen organizado debido a que la mayoría ingresa de manera ilegal o irregular. ${ }^{29}$

Bajo este orden de ideas, el Plan Integral de Desarrollo para Centroamérica se enfoca principalmente en la contención de la migración y en atender los problemas in situ. En este plan participan agencias de la ONU y la Organización Internacional de Migraciones (OIM), entre otros. Este plan inició con el programa sembrando vidas que entrega casi 5000 pesos mensuales, insumos y capacitación técnica a personas del sector agrícola y con el programa de jóvenes construyendo el futuro que ofrece oportunidades de empleo en empresas y talleres a individuos menores de 30 años y destina alrededor de 3748 pesos de manera mensual y seguro de vida a los participantes. ${ }^{30}$

Se pactó con los Estados Unidos una inversión de 800 millones de dólares, 400 se invertirán en una planta de gas natural licuado, 320 en la producción de

\footnotetext{
29 Louidor, Wooldy Edson, Introducción a los estudios migratorios: migraciones y derechos humanos en la era de la globalización, Bogotá, Pontificia Universidad Javeriana, 2017, p. 133

30 Noriega Curtis, Yalí y Barragán, Pedro Ludwig, "Una nueva realidad migratoria para Centroamérica", 3 de junio de 2021, https://revistafal.com/una-nueva-realidad-migratoria-para-centroamerica/.
} 
energía eólica y 80 en la pequeña y mediana empresa. ${ }^{31}$ Ahora bien, la Comisión Económica para América Latina y el Caribe (CEPAL) y la Organización de las Naciones Unidas (ONU) respaldaron este Plan Integral de Centroamérica. El programa Sembrando Vida en El Salvador ${ }^{32}$ tiene por objeto, contribuir a reducir la pobreza y la migración forzada en el campo. El requisito para recibir el apoyo es: ser mayor de edad, residente originario de municipios con flujo migratorio y tener tierra área sembrar. Serán beneficiadas 20 mil personas con 250 dólares mensuales. México otorgará 31 millones de dólares para la siembra de 50 mil hectáreas de árboles frutales y maderables. El plan de apoyo para Centroamérica es respaldado por 35 países, 65 agencias de cooperación y 8 organismos internacionales. En Honduras se beneficiarán 13,000 personas con 180 dólares mensuales. ${ }^{33}$ El objetivo del plan es construir una Centroamérica más próspera y segura para resolver las causas subyacentes de la migración a fin de que los ciudadanos de la región puedan tener una vida mejor en su país junto con sus familias.

El ocho de junio del 2021 se firmó un memorándum ${ }^{34}$ de entendimiento entre México y Estados Unidos con el objeto de lograr una Norteamérica próspera y segura basada en una sociedad justa y equitativa con oportunidades económicas y seguridad para todos. Los objetivos se enfocaron en fomentar la inversión en el sur de México, (incluidos préstamos para viviendas asequibles) y en fortalecer las cadenas de valor rurales de cacao, café y ecoturismo. Por otra parte, los Estados Unidos comprometieron alrededor de \$130 millones de dólares para los próximos tres años con el fin de apoyar la implementación de la reforma laboral de México. Otros compromisos firmados en el memorándum son: (i) desarticular las organizaciones de trata de personas; (ii) abordar las causas fundamentales de la migración en los países del Triángulo del Norte; (iii) compartir información y estrategias; (iv) administrar nuevos programas para fomentar las oportunidades económicas a través del desarrollo agrícola y el empoderamiento de los jóvenes; (v) aumentar la cooperación para proteger las fronteras y garantizar una inmigración ordenada.

\section{Conclusiones}

El endurecimiento de la política migratoria México-Estados Unidos durante el mandato del entonces presidente Donald Trump trajo como consecuencia que miles de migrantes de Centroamérica se vieran en la imperiosa necesidad de solicitar asilo en México debido a que bajo el programa Quédate en México los solicitantes de asilo a Estados Unidos tenían que permanecer en México mientras se resolvía su solicitud. El tema migratorio fue abordado por Trump como un

31 Butrón, Jorge, "Pacta EU 800 mdd para desarrollo del sur: SRE”, La Razón, 11 de julio de 2019, p. 9.

32 LóPEZ, Antonio, “Anuncian sembrando vida, pero aún no da frutos en El Salvador”, La Razón, 7 de octubre 2019, p. 3 .

33 "México pacta entrega directa de apoyos sociales a salvadoreños", La Razón, 9 de octubre de 2019, p. 3.

34 Secretaría de Relaciones Exteriores, "Informe para Senado de la República: visita de la vicepresidenta de Estados Unidos, Kamala D. Harris a la ciudad de México, 8 de junio de 2021", pp. 14-22, https://infosen. senado.gob.mx/sgsp/gaceta/64/3/2021-06-16-1/assets/documentos/RE_Informe_Visita_Kamala_Harris.pdf. 
asunto de seguridad nacional y la pandemia de COVID-19 contribuyó para que mandatario invocara el título 42 y deportara de manera inmediata a todas las personas que cruzaran la frontera de los Estados Unidos, so pretexto de emergencia sanitaria sin que mediara ningún tipo de procedimiento de deportación. Algunos migrantes optaron por quedarse en México debido a que el MPP fue suspendido durante la pandemia, otros, por su parte buscaron rutas terrestres o marítimas con un alto riesgo y costo para poder llegar a los Estados Unidos. Se puede decir que con la firma de la Declaración Conjunta de 7 de junio de 2019 y con la firma de acuerdos y convenios con los países del Triángulo del Norte, Trump logró la securitización de la migración y aumentó el nacionalismo en su país.

Con la llegada a la presidencia de Joe Biden, la retórica ha cambiado, sin embargo, las fronteras terrestres siguen cerradas y el título 42 se sigue aplicando. El programa Quédate en México finalizó el primero de junio del 2021, sin embargo, todavía faltan resoluciones pendientes bajo el MPP. Un logro importante es que el ocho de junio de 2021, México y Estados Unidos firmaron un memorándum de entendimiento en materia de cooperación internacional para atender las causas estructurales de la migración e impulsar el desarrollo económico de manera sustentable en el sur de México y norte de Centroamérica con el Plan Integral de Centroamérica avalado por la CEPAL y con programas como sembrando vidas y jóvenes construyendo el futuro. Como se comentó con antelación, el programa Sembrando vida otorga opciones laborales en el sector agrícola y evita la migración por razones económicas. Y el programa de jóvenes construyendo el futuro ayuda a ese sector a conseguir empleo mediante prácticas renumeradas. Estados Unidos se comprometió a aumentar su contribución económica para la implementación de la reforma laboral en México, pero desgraciadamente, la pandemia de COVID-19 ha durado más de lo previsto y ha empeorado la situación de miles de migrantes que viven en la pobreza, que temen por su vida y que buscan mejorar sus condiciones de vida. La cooperación internacional entre México, Estados Unidos y los países del Triángulo del Norte es indispensable para el desarrollo económico en la región y así evitar la migración forzada, porque la migración debe de ser un derecho no una necesidad.

\section{Bibliografía}

Aduanas y protección fronteriza de Estados Unidos, "Detenciones en la frontera", La Razón, 7 de enero de 2020.

Agenda 2030, "La Asamblea General adopta la Agenda 2030 para el Desarrollo Sostenible", https://www.un.org/sustainabledevelopment/es/2015/09/la-asamblea-general-adopta-la-agenda-2030-para-el-desarrollo-sostenible/.

Alto Comisionado de las Naciones Unidas para los Refugiados (ACNUR), Pacto Mundial sobre Refugiados, con una nota introductoria de la Oficina del Alto Comisionado de las Naciones Unidas para los Refugiados (ACNUR), 17 de diciembre de 2018, https://www.refworld.org.es/docid/5c781b294.html. 
BAUGH, Ryan, “Annual Flow Report: Refugees and Asylees 2019”, septiembre de 2020, https:/www.dhs.gov/sites/default/files/publications/immigration-statistics/yearbook/2019/refugee_and_asylee_2019.pdf.

Butrón, Jorge, "Pacta EU 800 md̄ para desarrollo del sur: SRE”, La Razón, 11 de julio de 2019.

COMAR, "Estadísticas de solicitantes de la condición de refugiado en México", marzo de 2020, https://www.gob.mx/comar/articulos/estadisticas-de-solicitantes-de-la-condicion-de-refugiado-en-mexico.

COMAR, "La COMAR en números”, mayo de 2021, https:/www.gob.mx/comar/ articulos/la-comar-en-numeros-273823?idiom=es.

COMAR, "Requisitos para solicitar Refugio en México", https://www.gob.mx/ comar.

Convención de Ginebra de 1951 sobre el estatuto de los refugiados y del protocolo de 1967, https://www.acnur.org/fileadmin/Documentos/BDL/2001/ 0506.pdf.

Contreras Delgado, Camilo; París Pombo, María Dolores y Velasco Ortiz, Laura (coords.), Caravanas Migrantes y Desplazamientos Colectivos en la Frontera México-Estados Unidos, México, El Colegio de la Frontera Norte, 2021.

Departamento de Seguridad Nacional, "Departamento de Seguridad Nacional (DHS) anuncia proceso para atender a individuos en México con casos pendientes bajo MPP", https://www.dhs.gov/news/2021/02/11/departamento-deseguridad-nacional-dhs-anuncia-proceso-para-atender-individuos-en-m\#.

Departamento de Seguridad Nacional, "Acuerdos del DHS con Guatemala, Honduras y el Salvador”, https:/www.dhs.gov/sites/default/files/publications/19_1007_fact-sheet-spanish-agreements-w-northern-region-central-amcountries.pdf.

Departamento de Seguridad Nacional, "Terminación del Programa de Protección al Migrante", 1 de junio de 2021,chttps://www.dhs.gov/sites/default/files/ publications/21_0601_cancillertermination_of_mpp_program.pdf.

Departamento de Segurida $\bar{d}$ Nacional, "Orientación sobre políticas para la implementación de los Protocolos de Protección al Migrante" (Policy guidance for implementation of the Migrant Protection Protocols), https://www.dhs. gov/sites/default/files/publications/19_0129_OPA_migrant-protection-protocols-policy-guidance.pdf.

Departamento de Seguridad Nacional, "Métricas y Medidas de los Protocolos de Protección a Migrantes", https:/www.dhs.gov/sites/default/files/publications/ metricas_y_medidas_de_los_protocolos_de_proteccion_a_migrantes_2.pdf. De HaAs Hein, Castles Stephen y Miller, Mark, The age of migration: International population movements in the modern world, 6a.ed., Nueva York, Guilford Publications, 2020.

Durand, Jorge, Historia mínima de la migración México-Estados Unidos, México, El Colegio de México, primera reimpresión, 2017. 
GutiéRrez GonzÁLEZ, Alicia, "Finaliza programa quédate en México", El Sol de México, 22 de junio de 2021, https:/www.elsoldemexico.com.mx/analisis/ finaliza-programa-quedate-en-mexico-6872525.html.

Instituto Nacional de Migración, "Trámites Migratorios", https://www.gob.mx/ inm/acciones-y-programas/tramites-migratorios.

Koser, Khalid, International Migration. A very short introduction, 2a. ed., Oxford, Oxford University Press, 2016.

La Razón, "México pacta entrega directa de apoyos sociales a salvadoreños", 9 de octubre de 2019.

Ley sobre Refugiados, Protección Complementaria y Asilo Político, del 27 de enero de 2011, http://www.diputados.gob.mx/LeyesBiblio/pdf/ LRPCAP_111120.pdf.

LóPEZ, Antonio, “Anuncian sembrando vida, pero aún no da frutos en El Salvador", La Razón, 7 de octubre 2019.

LouIDOR, Wooldy Edson, Introducción a los estudios migratorios: migraciones y derechos humanos en la era de la globalización, Bogotá, Pontificia Universidad Javeriana, 2017.

Nieto, Francisco, "Camino hacia EU", El Heraldo de México, 19 de junio de 2019, https://issuu.com/elheraldodemexico/docs/el_heraldo_771.

Noriega Curtis, Yalí y Barragán, Pedro Ludwig, "Uña nueva realidad migratoria para Centroamérica", 3 de junio de 2021, https:/revistafal.com/unanueva-realidad-migratoria-para-centroamerica/.

PÉREZ, Maritza, "Crecen $125 \%$ cruces hacia EU de migrantes mexicanos", El Economista, 9 de abril de 2021, https://www.eleconomista.com.mx/politica/ Crecen-125-cruces-hacia-EU-de-migrantes-mexicanos-20210409-0005.html. PInEDA, Perla, "Ebrard presentará informe migratorio", El Economista, 9 de septiembre de 2019.

Secretaría de Relaciones Exteriores, "Declaración Conjunta de 7 de junio de 2019”, https://www.gob.mx/sre/documentos/declaracion-conjunta-203701.

Secretaría de Relaciones Exteriores, "Intervención del secretario de Relaciones Exteriores de México, Marcelo Ebrard. Conferencia Intergubernamental para la adopción del Pacto Mundial para la Migración Segura, Ordenada y Regular, Sesión plenaria", Marruecos, 10 de diciembre de 2018, https://www. un.org/en/conf/migration/assets/pdf/GCM-Statements/mexico.pdf.

Secretaría de Relaciones Exteriores, "Informe para el Senado de la República: visita de la vicepresidenta de Estados Unidos, Kamala d. Harris a la ciudad de México, 8 de junio de 2021", https://infosen.senado.gob.mx/sgsp/gaceta/64/3/2021-06-16-1/assets/documentos/RE_Informe_Visita_Kamala_Harris.pdf.

U.S. Customs and Border Protection, "Nationwide Enforcement Encounters: Title 8 Enforcement Actions and Title 42 Expulsions", https://www.cbp.gov/ newsroom/stats/cbp-enforcement-statistics/title-8-and-title-42-statistics. 